\title{
X.500 Lightweight Directory Access Protocol
}

Status of this Memo

This RFC specifies an IAB standards track protocol for the Internet community, and requests discussion and suggestions for improvements. Please refer to the current edition of the "IAB Official Protocol Standards" for the standardization state and status of this protocol. Distribution of this memo is unlimited.

\section{Abstract}

The protocol described in this document is designed to provide access to the Directory while not incurring the resource requirements of the Directory Access Protocol (DAP). This protocol is specifically targeted at simple management applications and browser applications that provide simple read/write interactive access to the Directory, and is intended to be a complement to the DAP itself.

Key aspects of LDAP are:

- Protocol elements are carried directly over TCP or other transport, bypassing much of the session/presentation overhead.

- Many protocol data elements are encoding as ordinary strings (e.g., Distinguished Names).

- A lightweight BER encoding is used to encode all protocol elements.

\section{History}

The tremendous interest in X.500 [1,2] technology in the Internet has lead to efforts to reduce the high "cost of entry" associated with use of the technology, such as the Directory Assistance Service [3] and DIXIE [4]. While efforts such as these have met with success, they have been solutions based on particular implementations and as such have limited applicability. This document continues the efforts to define Directory protocol alternatives but departs from previous efforts in that it consciously avoids dependence on particular 
implementations.

2. Protocol Model

The general model adopted by this protocol is one of clients performing protocol operations against servers. In this model, this is accomplished by a client transmitting a protocol request describing the operation to be performed to a server, which is then responsible for performing the necessary operations on the Directory. Upon completion of the necessary operations, the server returns a response containing any results or errors to the requesting client. In keeping with the goal of easing the costs associated with use of the Directory, it is an objective of this protocol to minimize the complexity of clients so as to facilitate widespread deployment of applications capable of utilizing the Directory.

Note that, although servers are required to return responses whenever such responses are defined in the protocol, there is no requirement for synchronous behavior on the part of either client or server implementations: requests and responses for multiple operations may be exchanged by client and servers in any order, as long as clients eventually receive a response for every request that requires one.

Consistent with the model of servers performing protocol operations on behalf of clients, it is also to be noted that protocol servers are expected to handle referrals without resorting to the return of such referrals to the client. This protocol makes no provisions for the return of referrals to clients, as the model is one of servers ensuring the performance of all necessary operations in the Directory, with only final results or errors being returned by servers to clients.

Note that this protocol can be mapped to a strict subset of the directory abstract service, so it can be cleanly provided by the DAP.

3. Mapping Onto Transport Services

This protocol is designed to run over connection-oriented, reliable transports, with all 8 bits in an octet being significant in the data stream. Specifications for two underlying services are defined here, though others are also possible.

\subsection{Transmission Control Protocol (TCP)}

The LDAPMessage PDUs are mapped directly onto the TCP bytestream. Server implementations running over the TCP should provide a protocol listener on port 389 . 


\subsection{Connection Oriented Transport Service (COTS)}

The connection is established. No special special use of T-Connect is made. Each LDAPMessage PDU is mapped directly onto T-Data.

4. Elements of Protocol

For the purposes of protocol exchanges, all protocol operations are encapsulated in a common envelope, the LDAPMessage, which is defined as follows:

LDAPMessage : := SEQUENCE \{ messageID MessageID, protocolop CHOICE \{

bindRequest BindRequest, bindResponse BindResponse, unbindRequest UnbindRequest searchRequest SearchRequest, searchResponse SearchResponse, modifyRequest ModifyRequest, modifyResponse ModifyResponse, addRequest AddRequest, addResponse AddResponse, delRequest delResponse DelRequest, modifyRDNRequest ModifyRDNRequest, modifyRDNResponse ModifyRDNResponse, compareDNRequest CompareRequest, compareDNResponse CompareResponse, abandonRequest AbandonRequest

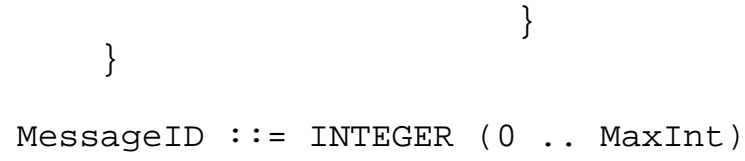
common fields required in all protocol exchanges. At this time the only common field is a message ID, which is required to have a value different from the values of any other requests outstanding in the LDAP session of which this message is a part.

The message ID value must be echoed in all LDAPMessage envelopes encapsulting responses corresponding to the request contained in the LDAPMessage in which the message ID value was originally used.

In addition to the LDAPMessage defined above, the following definitions are also used in defining protocol operations: 
IA5string : := OCTET STRING

The IA5string is a notational convenience to indicate that, although strings of IA5String type encode as OCTET STRING types, the legal character set in such strings is limited to the IA5 character set.

LDAPDN : := IA5string

RelativeLDAPDN ::= IA5string

An LDAPDN and a RelativeLDAPDN are respectively defined to be the representation of a Distinguished Name and a Relative Distinguished Name after encoding according to the specification in [5], such that

<distinguished-name> : := <name>

<relative-distinguished-name> : := <name-component>

where <name> and <name-component> are as defined in [5].

AttributeValueAssertion : := SEQUENCE \{ attributeType AttributeType attributeValue AttributeValue \}

The AttributeValueAssertion type definition is similar to the one in the Directory standards.

AttributeType : := IA5string

AttributeValue : := OCTET STRING

An AttributeType value takes on as its value the textual string associated with that AttributeType in the Directory standards. For example, the AttributeType 'organizationName' with object identifier 2.5.4.10 is represented as an AttributeType in this protocol by the string "organizationName". In the event that a protocol

implementation encounters an Attribute Type with which it cannot associate a textual string, an ASCII string encoding of the object identifier associated with the Attribute Type may be subsitituted. For example, the organizationName AttributeType may be represented by the ASCII string "2.5.4.10" if a protocol implementation is unable to associate the string "organizationName" with it.

A field of type AttributeValue takes on as its value an octet string encoding of a Directory AttributeValue type. The definition of these string encodings for different Directory AttributeValue types may be 
found in companions to this document that define the encodings of various attribute syntaxes such as [6].

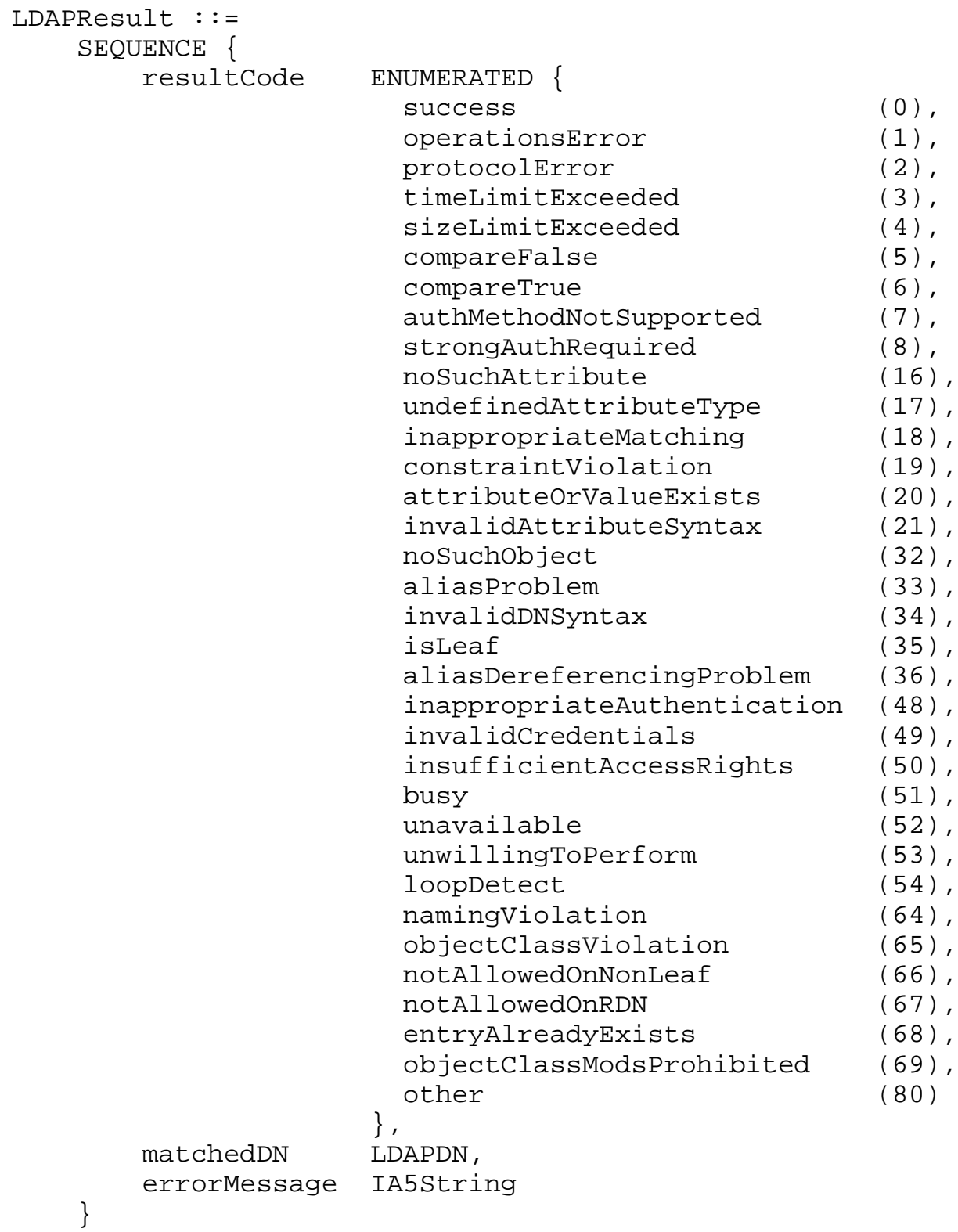

The LDAPResult is the construct used in this protocol to return success or failure indications from servers to clients. In response to various requests, servers will return responses containing fields 
of type LDAPResult to indicate the final status of a protocol operation request. The errorMessage field of this construct may, at the servers option, be used to return an ASCII string containing a textual, human-readable error diagnostic. As this error diagnostic is not standardized, implementations should not rely on the values returned. If the server chooses not to return a textual diagnostic, the errorMessage field of the LDAPResult type should contain a zero length string.

For resultCodes of noSuchobject, aliasProblem, invalidDNSyntax, isleaf, and aliasDereferencingProblem, the matchedDN field is set to the name of the lowest entry (object or alias) in the DIT that was matched and is a truncated form of the name provided or, if an alias has been dereferenced, of the resulting name. The matchedDN field should be set to NULL DN (a zero length string) in all other cases.

\subsection{Bind Operation}

The function of the Bind Operation is to initiate a protocol session between a client and a server, and to allow the authentication of the client to the server. The Bind Operation must be the first operation request received by a server from a client in a protocol session. The Bind Request is defined as follows:

BindRequest : : = [APPLICATION 0] SEQUENCE \{ version INTEGER (1 .. 127), name LDAPDN, authentication CHOICE \{ simple [0] OCTET STRING, krbv42LDAP [1] OCTET STRING, \} krbv42DSA [2] OCTET STRING

\}

Parameters of the Bind Request are:

- version: A version number indicating the version of the protocol to be used in this protocol session. This document describes version 2 of the LDAP protocol. Note that there is no version negotiation, and the client should just set this parameter to the version it desires.

- name: The name of the Directory object that the client wishes to bind as. This field may take on a null value (a zero length string) for the purposes of anonymous binds.

- authentication: information used to authenticate the name, if any, 
provided in the Bind Request. The "simple" authentication option provides minimal authentication facilities, with the contents of the authentication field consisting only of a cleartext password. This option should also be used when unauthenticated or anonymous binds are to be performed, with the field containing a zero length string in such cases. Kerberos version 4 [7] authentication to the LDAP server and the DSA is accomplished by using the "krbv42LDAP" and "krbv42DSA" authentication options, respectively. Note that though they are referred to as separate entities here, there is no requirement these two entities be distinct (i.e., a DSA could speak LDAP directly). Two separate authentication options are provided to support all implementations. Each octet string should contain the kerberos ticket (e.g., as returned by krb_mk_req()) for the appropriate service. The suggested service name for authentication to the LDAP server is "ldapserver". The suggested service name for authentication to the DSA is "x500dsa". In both cases, the suggested instance name for the service is the name of the host on which the service is running. Of course, the actual service names and instances will depend on what is entered in the local kerberos principle database.

The Bind Operation requires a response, the Bind Response, which is defined as:

BindResponse : := [APPLICATION 1] LDAPResult

A Bind Response consists simply of an indication from the server of the status of the client's request for the initiation of a protocol session.

Upon receipt of a Bind Request, a protocol server will authenticate the requesting client if necessary, and attempt to set up a protocol session with that client. The server will then return a Bind Response to the client indicating the status of the session setup request.

\subsection{Unbind Operation}

The function of the Unbind Operation is to terminate a protocol session. The Unbind Operation is defined as follows:

UnbindRequest $::=[$ APPLICATION 2] NULL

The Unbind Operation has no response defined. Upon transmission of an UnbindRequest, a protocol client may assume that the protocol session is terminated. Upon receipt of an UnbindRequest, a protocol server may assume that the requesting client has terminated the session and that all outstanding requests may be discarded. 


\subsection{Search Operation}

The Search Operation allows a client to request that a search be performed on its behalf by a server. The search Request is defined as follows :

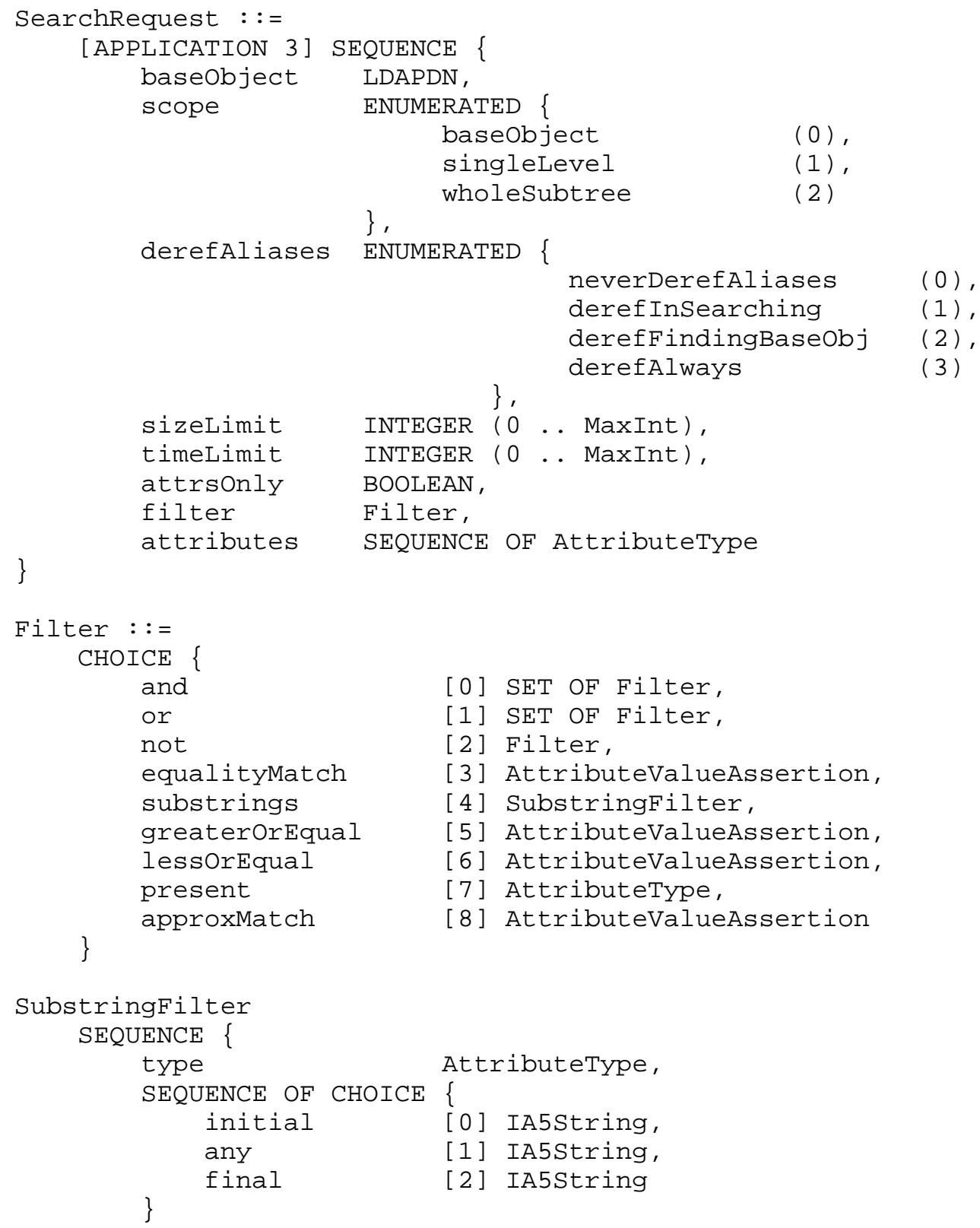

Yeong, Howes \& Kille

[Page 8] 
Parameters of the Search Request are:

- baseObject: An LDAPDN that is the base object entry relative to which the search is to be performed.

- scope: An indicator of the scope of the search to be performed. The semantics of the possible values of this field are identical to the semantics of the scope field in the Directory Search Operation.

- derefAliases: An indicator as to how alias objects should be handled in searching. The semantics of the possible values of this field are, in order of increasing value:

neverDerefAliases: do not dereference aliases in searching or in locating the base object of the search;

derefInSearching: dereference aliases in subordinates of the base object in searching, but not in locating the base object of the search;

dereffindingBaseobject: dereference aliases in locating the base object of the search, but not when searching subordinates of the base object;

derefAlways: dereference aliases both in searching and in locating the base object of the search.

- sizelimit: A sizelimit that restricts the maximum number of entries to be returned as a result of the search. A value of 0 in this field indicates that no sizelimit restrictions are in effect for the search.

- timelimit: A timelimit that restricts the maximum time (in seconds) allowed for a search. A value of 0 in this field indicates that no timelimit restrictions are in effect for the search.

- attrsonly: An indicator as to whether search results should contain both attribute types and values, or just attribute types. Setting this field to TRUE causes only attribute types (no values) to be returned. Setting this field to FALSE causes both attribute types and values to be returned.

- filter: A filter that defines the conditions that must be fulfilled in order for the search to match a given entry.

- attributes: A list of the attributes from each entry found as a 
result of the search to be returned. An empty list signifies that all attributes from each entry found in the search are to be returned.

The results of the search attempted by the server upon receipt of a Search Request are returned in Search Responses, defined as follows:

Search Response : := CHOICE entry

[APPLICATION 4] SEQUENCE \{ objectName LDAPDN, attributes SEQUENCE OF SEQUENCE \{ AttributeType, \} SET OF AttributeValue

\} , resultcode [APPLICATION 5] LDAPResult

Upon receipt of a Search Request, a server will perform the necessary search of the DIT.

The server will return to the client a sequence of responses comprised of:

- Zero or more Search Responses each consisting of an entry found during the search; with the response sequence terminated by

- A single search Response containing an indication of success, or detailing any errors that have occurred.

Each entry returned will contain all attributes, complete with associated values if necessary, as specified in the 'attributes' field of the Search Request.

Note that an X.500 "list" operation can be emulated by a one-level LDAP search operation with a filter checking for the existence of the objectclass attribute, and that an X.500 "read" operation can be emulated by a base object LDAP search operation with the same filter.

\subsection{Modify Operation}

The Modify Operation allows a client to request that a modification of the DIB be performed on its behalf by a server. The Modify Request is defined as follows: 


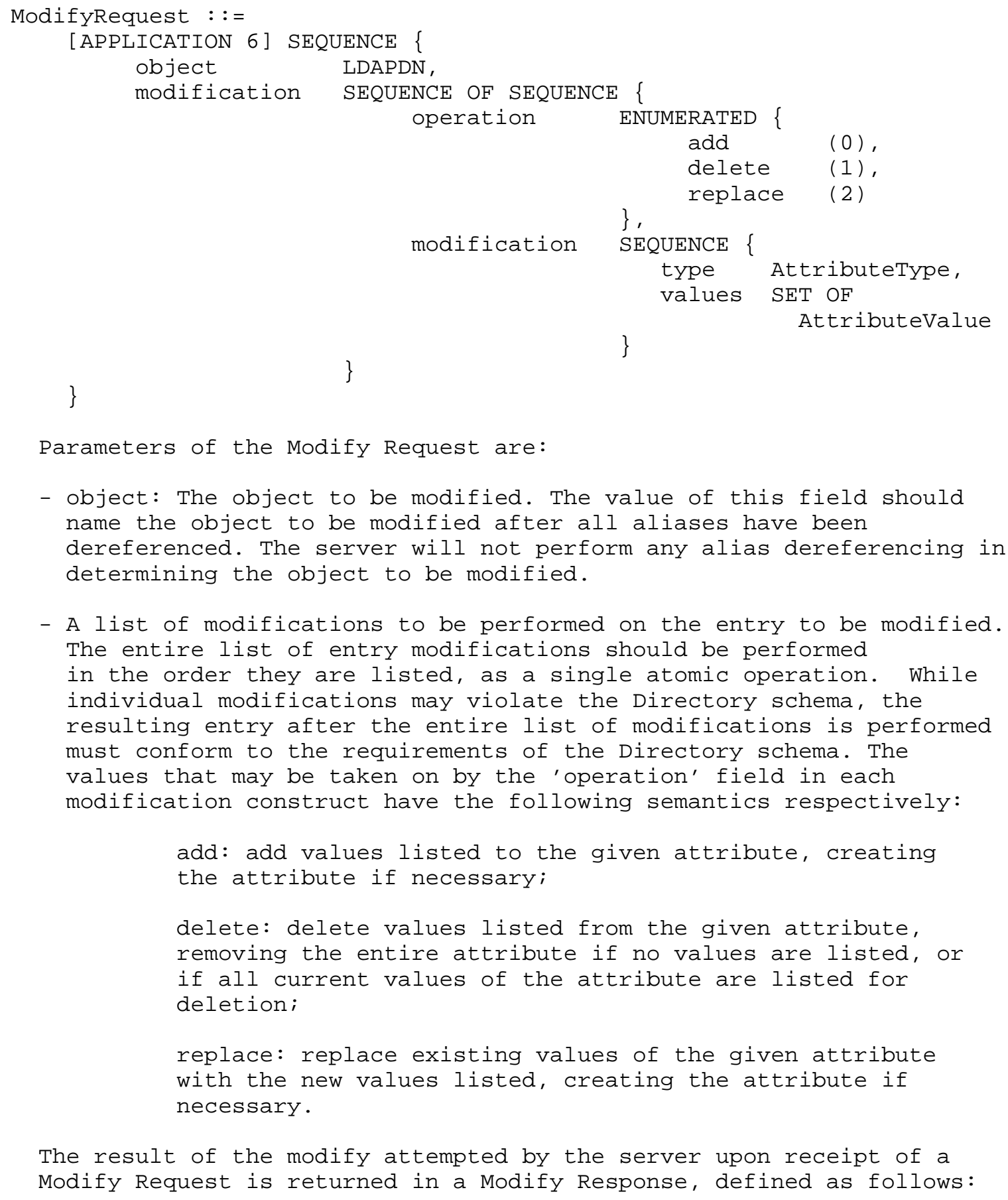

- A list of modifications to be performed on the entry to be modified. The entire list of entry modifications should be performed in the order they are listed, as a single atomic operation. While individual modifications may violate the Directory schema, the resulting entry after the entire list of modifications is performed must conform to the requirements of the Directory schema. The values that may be taken on by the 'operation' field in each modification construct have the following semantics respectively:

add: add values listed to the given attribute, creating the attribute if necessary;

delete: delete values listed from the given attribute, removing the entire attribute if no values are listed, or if all current values of the attribute are listed for deletion;

replace: replace existing values of the given attribute with the new values listed, creating the attribute if necessary.

The result of the modify attempted by the server upon receipt of a Modify Request is returned in a Modify Response, defined as follows: 


\section{ModifyResponse : := [APPLICATION 7] LDAPResult}

Upon receipt of a Modify Request, a server will perform the necessary modifications to the DIB.

The server will return to the client a single Modify Response indicating either the successful completion of the DIB modification, or the reason that the modification failed. Note that due to the requirement for atomicity in applying the list of modifications in the Modify Request, the client may expect that no modifications of the DIB have been performed if the Modify Response received indicates any sort of error, and that all requested modifications have been performed if the Modify Response indicates successful completion of the Modify Operation.

\subsection{Add Operation}

The Add Operation allows a client to request the addition of an entry into the Directory. The Add Request is defined as follows:

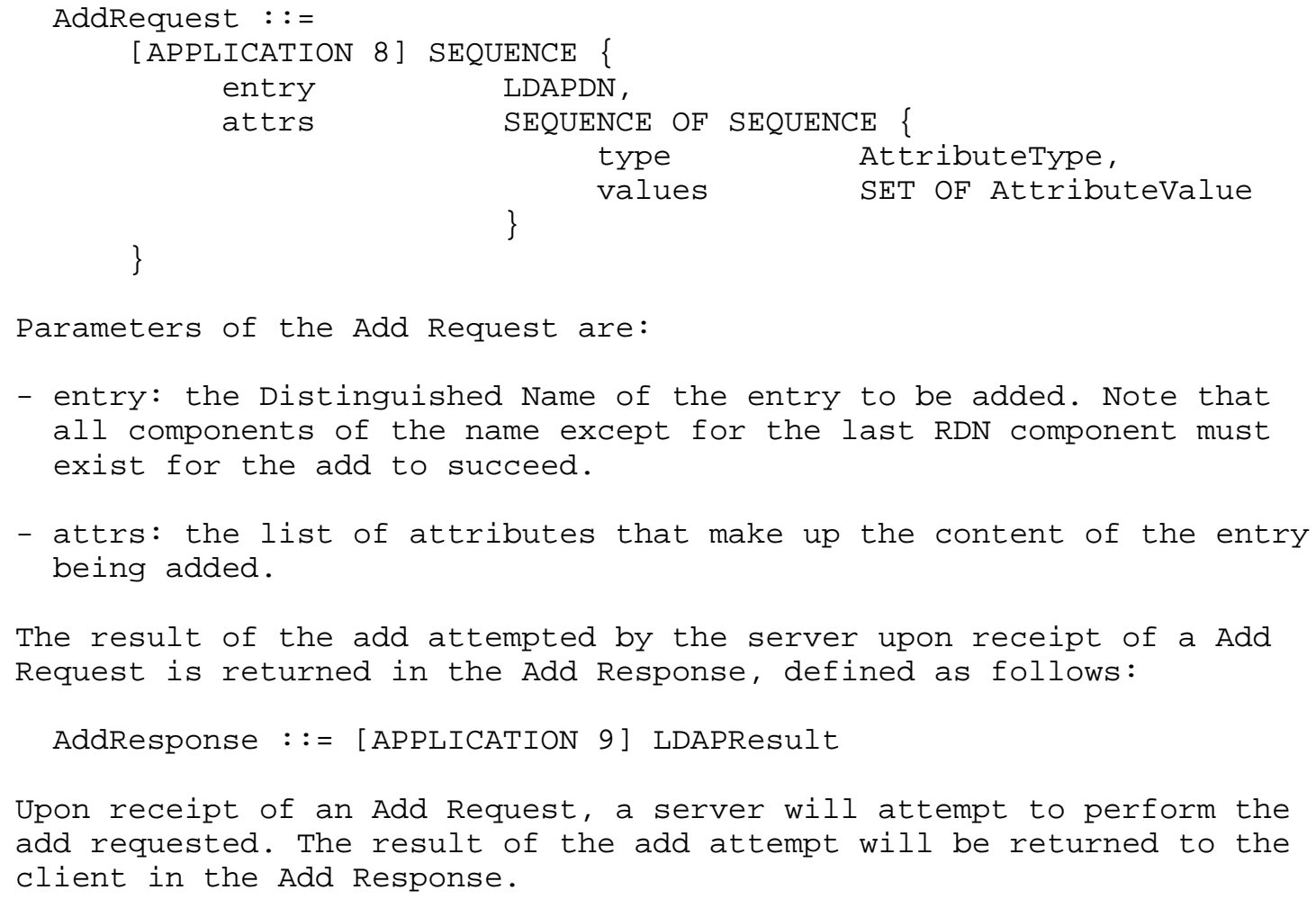


4.6. Delete Operation

The Delete Operation allows a client to request the removal of an entry from the Directory. The Delete Request is defined as follows:

DelRequest : := [APPLICATION 10] LDAPDN

The Delete Request consists only of the Distinguished Name of the entry to be deleted. The result of the delete attempted by the server upon receipt of a Delete Request is returned in the Delete Response, defined as follows:

DelResponse : := [APPLICATION 11] LDAPResult

Upon receipt of a Delete Request, a server will attempt to perform the entry removal requested. The result of the delete attempt will be returned to the client in the Delete Response. Note that only leaf objects may be deleted with this operation.

\subsection{Modify RDN Operation}

The Modify RDN Operation allows a client to change the last component of the name of an entry in the Directory. The Modify RDN Request is defined as follows:

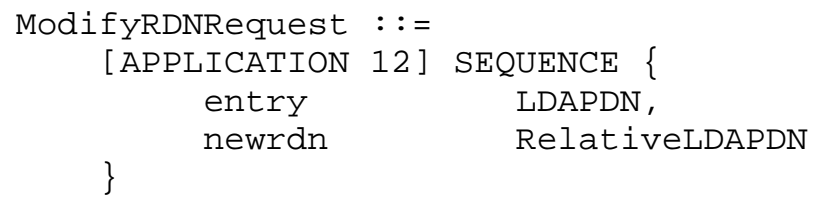

Parameters of the Modify RDN Request are:

- entry: the name of the entry to be changed.

- newrdn: the RDN that will form the last component of the new name.

The result of the name change attempted by the server upon receipt of a Modify RDN Request is returned in the Modify RDN Response, defined as follows:

ModifyRDNResponse : := [APPLICATION 13] LDAPResult

Upon receipt of a Modify RDN Request, a server will attempt to perform the name change. The result of the name change attempt will be returned to the client in the Modify RDN Response. The attributes that make up the old RDN are deleted from the entry. 
4.8. Compare Operation

The Compare Operation allows a client to compare an assertion provided with an entry in the Directory. The Compare Request is defined as follows:

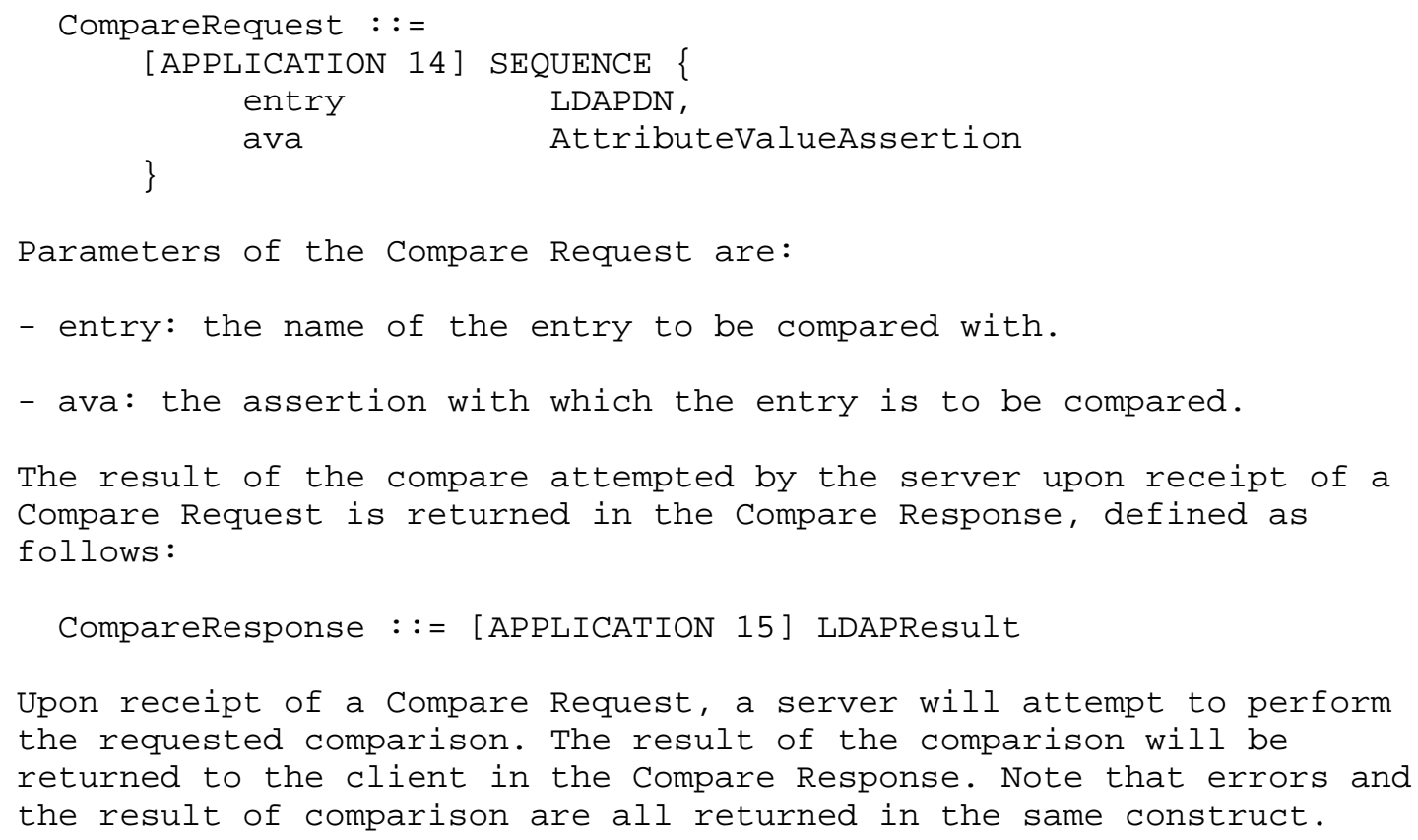

\subsection{Abandon Operation}

The function of the Abandon Operation is to allow a client to request that the server abandon an outstanding operation. The Abandon Request is defined as follows:

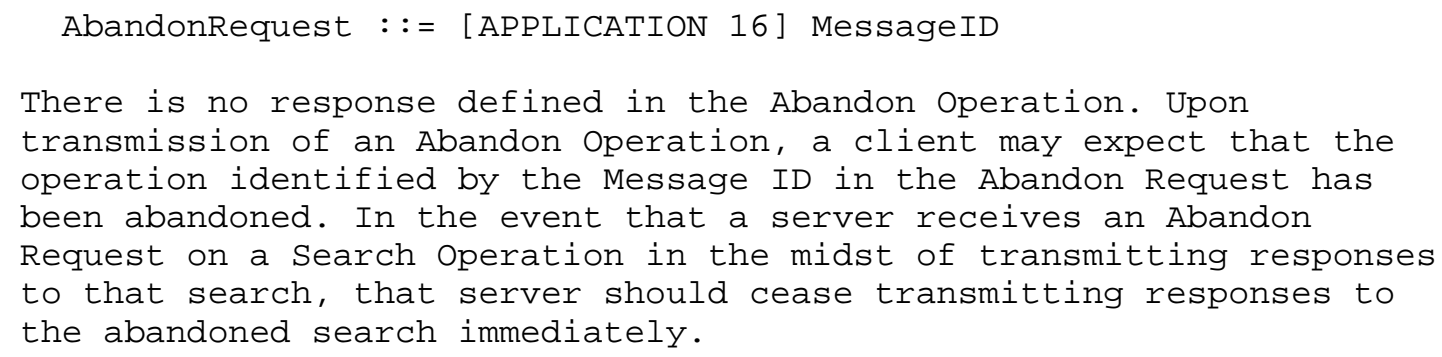




\section{Protocol Element Encodings}

The protocol elements of LDAP are encoded for exchange using the Basic Encoding Rules (BER) [11] of ASN.1 [10]. However, due to the high overhead involved in using certain elements of the BER, the following additional restrictions are placed on BER-encodings of LDAP protocol elements:

(1) Only the definite form of length encoding will be used.

(2) Bitstrings and octet strings will be encoded in the primitive form only.

6. Security Considerations

This version of the protocol provides facilities only for simple authentication using a cleartext password, and for kerberos version 4 authentication. Future versions of LDAP will likely include support for other authentication methods.

7. Bibliography

[1] The Directory: Overview of Concepts, Models and Service. CCITT Recommendation X.500, 1988 .

[2] Information Processing Systems -- Open systems Interconnection -The Directory: Overview of Concepts, Models and Service. ISO/IEC JTC 1/SC21; International Standard 9594-1, 1988.

[3] Rose, M., "Directory Assistance Service", RFC 1202, Performance Systems International, Inc., February 1991.

[4] Howes, R., Smith, M., and B. Beecher, "DIXIE Protocol Specification", RFC 1249, University of Michigan, August 1991.

[5] Kille, S., "A String Representation of Distinguished Names", RFC 1485, ISODE Consortium, July 1993.

[6] Howes, T., Kille, S., Yeong, W., and C. Robbins, "The String Representation of Standard Attribute Syntaxes", RFC 1488, University of Michigan, ISODE Consortium, Performance Systems International, NeXor Ltd., July 1993.

[7] Kerberos Authentication and Authorization System. S.P. Miller, B.C. Neuman, J.I. Schiller, J.H. Saltzer; MIT Project Athena Documentation Section E.2.1, December 1987. 
[8] The Directory: Models. CCITT Recommendation X.501 ISO/IEC JTC 1/SC21; International Standard 9594-2, 1988.

[9] The Directory: Abstract Service Definition. CCITT Recommendation X.511, ISO/IEC JTC 1/SC21; International Standard 9594-3, 1988.

[10] Specification of Abstract Syntax Notation One (ASN.1). CCITT Recommendation X.208, 1988 .

[11] Specification of Basic Encoding Rules for Abstract Syntax Notation One (ASN.1). CCITT Recommendation X.209, 1988.

9. Security Considerations

Security issues are not discussed in this memo.

9. Authors' Addresses

Wengyik Yeong

PSI, Inc.

510 Huntmar Park Drive

Herndon, VA 22070

USA

Phone: +1 703-450-8001

EMail: yeongw@psilink.com

Tim Howes

University of Michigan

ITD Research Systems

535 W William St.

Ann Arbor, MI 48103-4943

USA

Phone: +1 313 747-4454

EMail: timeumich.edu

Steve Kille

ISODE Consortium

PO Box 505

London

SW11 1DX

UK

Phone: +44-71-223-4062

EMail: S.Kille@isode.com 
Appendix A

Complete ASN.1 Definition

Lightweight-Directory-Access-Protocol DEFINITIONS : :=

IMPLICIT TAGS

BEGIN

LDAPMessage : := SEQUENCE \{

messageID MessageID,

-- unique id in request,

- to be echoed in response ( $\mathrm{s}$ )

protocolop

CHOICE \{

searchRequest SearchRequest, searchResponse SearchResponse, modifyRequest ModifyRequest, modifyResponse ModifyResponse, addRequest AddRequest, addResponse AddResponse, delRequest DelRequest, delResponse DelResponse, modifyDNRequest ModifyDNRequest, modifyDNResponse ModifyDNResponse, compareDNRequest CompareRequest, compareDNResponse CompareResponse, bindRequest BindRequest, bindResponse BindResponse, abandonRequest AbandonRequest, \} unbindRequest UnbindRequest

\}

BindRequest : :=

[APPLICATION 0] SEQUENCE \{

version INTEGER (1 .. 127),

name LDAPDN,

-- null name implies an anonymous bind

authentication CHOICE \{

simple [0] OCTET STRING, -- a zero length octet string

-- implies an unauthenticated

- bind.

krbv42LDAP [1] OCTET STRING,

krbv42DSA [2] OCTET STRING 


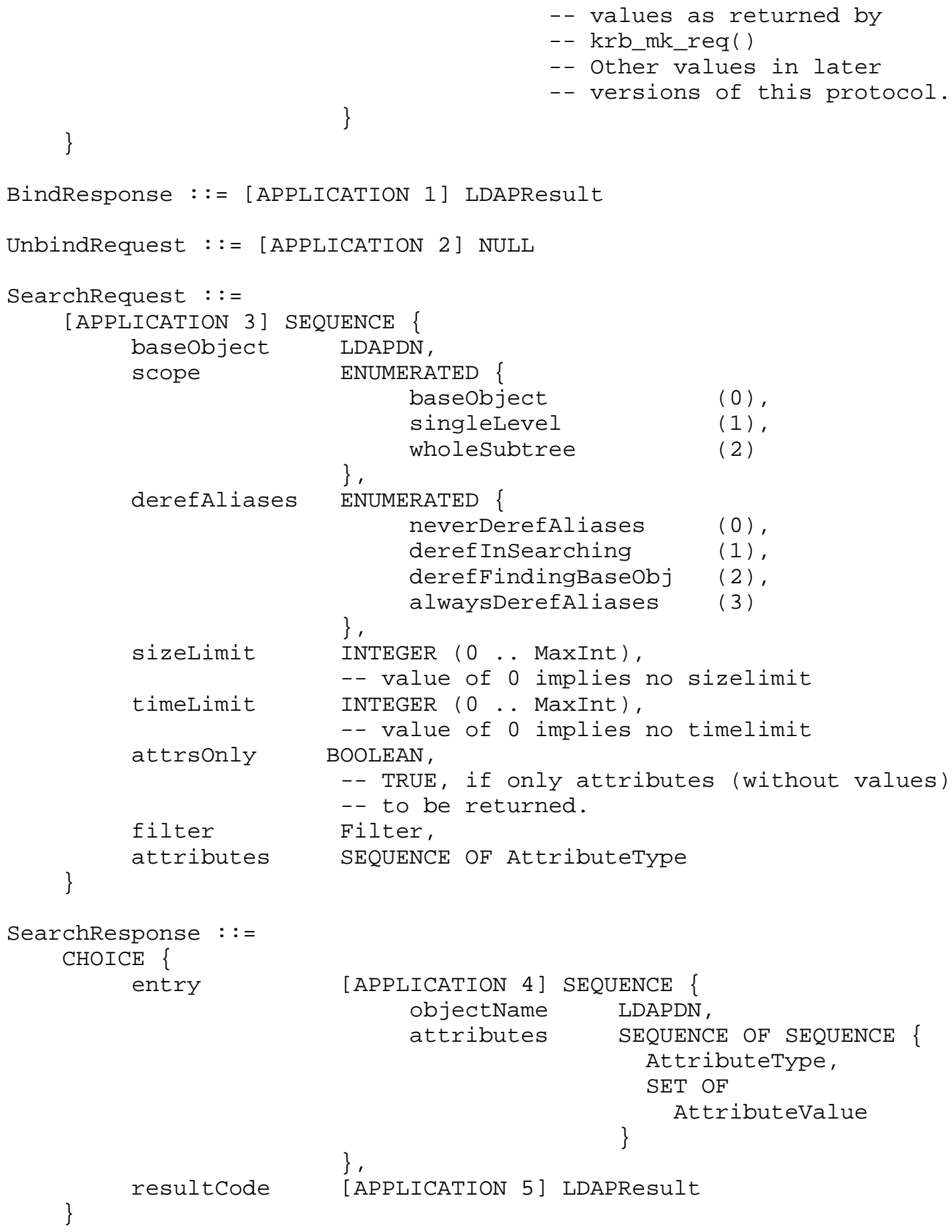




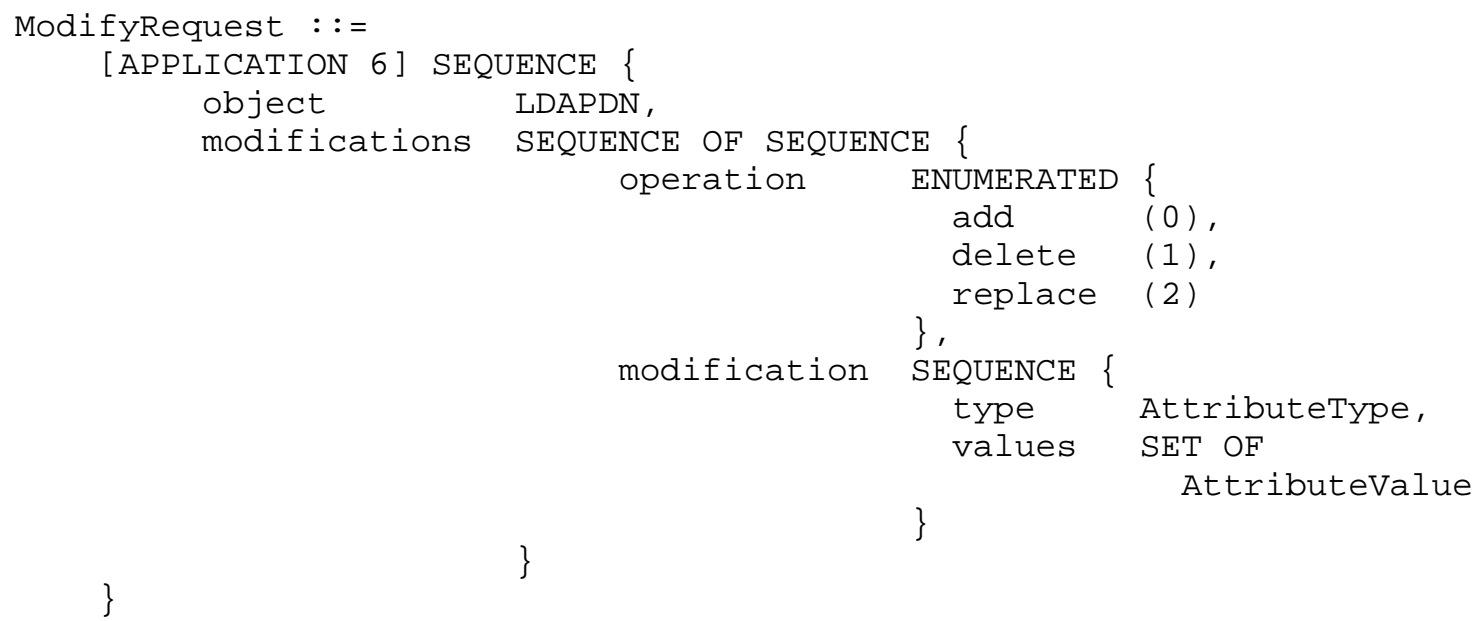


CompareResponse : := [APPLICATION 15] LDAPResult

AbandonRequest : : = [APPLICATION 16] MessageID

MessageID $::=$ INTEGER $(0 \ldots$ MaxInt $)$

LDAPDN : := IA5String

RelativeLDAPDN : := IA5String

Filter : :=

CHOICE \{
and
[0] SET OF Filter,
or
[1] SET OF Filter,
not
[2] Filter,
equalityMatch
[3] AttributeValueAssertion,
substrings
[4] SubstringFilter,
greaterorEqual [5] AttributeValueAssertion, lessOrEqual
present
[6] AttributeValueAssertion,
[7] AttributeType,
[8] AttributeValueAssertion

\}

LDAPResult : :=

SEQUENCE \{

resultcode

$\begin{array}{ll}\text { ENUMERATED \{ } & (0), \\ \text { success } & (1), \\ \text { operationsError } & (2), \\ \text { protocolError } & (3), \\ \text { timeLimitExceeded } & (4), \\ \text { sizeLimitExceeded } & (5), \\ \text { compareFalse } & (6), \\ \text { compareTrue } & (7), \\ \text { authMethodNotSupported } & (8), \\ \text { strongAuthRequired } & (16), \\ \text { noSuchAttribute } & (17), \\ \text { undefinedAttributeType } & (18), \\ \text { inappropriateMatching } & (19), \\ \text { constraintViolation } & (20), \\ \text { attributeOrValueExists } & (21), \\ \text { invalidAttributeSyntax } & (32), \\ \text { noSuchobject } & (33), \\ \text { aliasProblem } & (34), \\ \text { invalidDNSyntax } & (35), \\ \text { isLeaf } & (36), \\ \text { aliasDereferencingProblem } & (48), \\ \text { inappropriateAuthentication } & (49), \\ \text { invalidCredentials } & \end{array}$

Yeong, Howes \& Kille

[Page 20] 


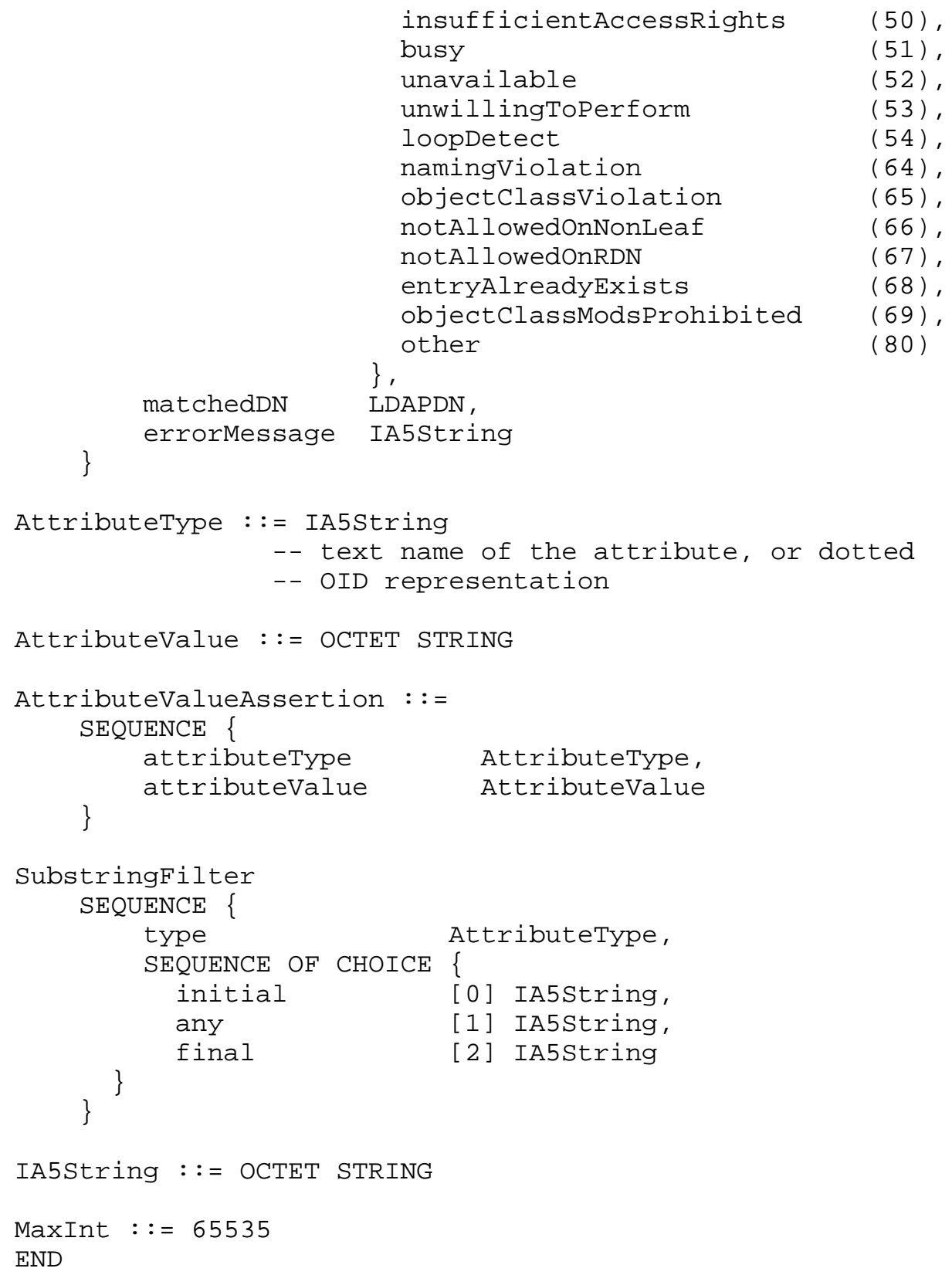

